\title{
Cars, compositionality, and consciousness
}

\section{Frederic Kaplan*}

Ecole Polytechnique Fédérale de Lausanne, Lausanne, Switzerland

*Correspondence: frederic.kaplan@epfl.ch

\section{A commentary on}

On the interactions between top-down anticipation and bottom-up regression

by Jun Tani

Learning to drive a car is a long and tedious process. For most of us, it takes many painful hours of training. At the beginning, the car is an external device, reacting in unpredictable ways. But progressively, we get used to some of the dynamics of the complex machine. We have to simultaneously learn basic control operations like switching the gears and pushing the brake, and to combine the operations into higher-level behavioral chunks like starting, stopping, turning, parking, and passing another car. Day after day we get used to the car's space occupation and the time necessary to slow down. The car becomes like our second skin. We learn to adapt each skill to specific contexts such as starting on a steep street, turning on a wet road, passing a long truck or parking in a busy Parisian boulevard. Based on the chunks we can easily anticipate and look ahead. We plan long trips by just focusing on important direction decisions and assuming that all the rest should run smoothly. For those who commute everyday by car, some driving trips are so familiar that the chunks can be done with almost no conscious control. Driving has become as natural as walking: an unconscious experience. However, as in walking, if something unexpected happens (e.g. a child crossing the street and a strange behavior of a car due to a flat tire) we are always ready to take control again and rapidly adapt to the new situation.

Tani (2007) doesn't write about cars. However, the article gives precious insights on how neural circuits can perform similar to the process we just described. It shows very convincingly how certain kinds of recurrent neural networks can acquire compositionality without using symbols. Compositionality is the property of systems that can be decomposed into reusable parts. Linguistic systems are typical examples of compositional systems. Discourses are combinations of sentences that are combination of words, which are combination of phonemes. Most sensorimotor behaviors, such as driving, are also intrinsically compositional, reusing basic primitives, chunks or schemas. It seems to be a fundamental feature for achieving complexity.

Compositional systems are very common in symbolic artificial intelligence. In these models, each chunk is an explicit structure that can be easily manipulated, organized in hierarchies and used for building plans. Unfortunately, most of the symbolic systems have difficulties in dealing with real-world dynamics for which plans must be constantly reassessed and the chunks must be continuously adapted to deal with the present situation. Tani's neural model not only shows that a dynamical system can exhibit compositional properties without having an explicit notion of chunk or schema, but also convincingly demonstrates that it can deal with complex realwork interaction in real time. The neural network has to discover by itself how to segment the continuous sensorimotor flow into sequences of reusable segments and to simultaneously arrange the chunks into higher-level sequences. The challenge is that both the processes have to run concurrently as in any real-world learning. The cornerstone of Tani's model is that it combines dynamic interactions between top-down look-ahead anticipations and a bottom regression process, permitting to re-situate the system if something goes wrong in the prediction. It is a wonderful illustration of self-organization at work.

There is something more. Tani's model tells us something important about the conscious experience associated with such compositional and dynamic management of behavior. Continuing some earlier discussions about the link between this kind of neural models and the conscious appraisal of time (Tani 2004), Tani suggests that when anticipation successively predicts the sensorimotor flow during the execution of a familiar behavioral sequence (e.g. driving on a familiar road), there is nothing to be "conscious" about. However, when a "breakdown" occurs, i.e., when there is a gap between the anticipation of the topdown system and the actual sensorimotor flow (e.g. a child crossing the street), the system has to perform intensive regression, which is an adjustment of the "self". This bottom-up process may result in the conscious experience associated with the unexpected event.

Compositionality and consciousness are likely to be intrinsically linked. The experience of driving a car is made of continuous shifts between unconscious "incorporated" sequences and small moments of awareness. To understand these bottom-up and topdown dynamics, we need plausible neural models of the emergence of compositional behavior. Tani's neurorobotic experiments are important steps in this direction.

\section{REFERENCES}

Tani, J. (2004). The dynamical systems accounts for phenomenology of immanent time. An interpretation by revisiting a robotics synthetic study. J. Conscious. Stud. 11, 5-24.

Tani, J. (2007). On the interactions between top-down anticipation and bottom-up regression. Front Neurorobotics. 1, 2.

Received: 16 October 2008; published: 15 December 2008

Citation: Front. Neurosci. (2008) 2, 2: 137. doi: 10.3389/ neuro.01.043.2008

Copyright: (c) 2008 Kaplan. This is an open-access publication subject to an exclusive license agreement between the authors and the Frontiers Research Foundation, which permits unrestricted use, distribution, and reproduction in any medium, provided the original authors and source are credited. 